\title{
Association of diabetes mellitus and structural changes in the central nervous system in children and adolescents: a systematic review
}

\author{
Ata pourabbasi ${ }^{1}$, Mehdi Tehrani-Doost ${ }^{2}$, Sogra Ebrahimi Qavam³ ${ }^{3}$ Seyed Masoud Arzaghi ${ }^{4}$ and Bagher Larijani ${ }^{5^{*}}$
}

\begin{abstract}
Background: The relationship between diabetes and academic performance have been of great interest to researchers during the year to date. Many studies have been conducted to discover this relationship during three recent decades. But, evaluation of the structural changes of brain in the context of diabetes is of paramount importance especially in children and adolescents.

Methods: This study is a systematic review conducted to investigate the structural changes in the central nervous system in children and adolescents living with diabetes. Among about 500 papers published in this area in Pubmed and SCOPUS, 13 articles in the field of assessing structural changes in the central nervous system in children and adolescents with diabetes mellitus were entered into the evaluation process.

Results: As can be seen in these studies, a huge proportion of structures of the central nervous system have been affected by diabetes that include different areas of gray and white matters. In the majority of these studies, it has become clear that high glycemic changes, especially recurrent hyperglycemic attacks are very seriously associated with structural changes in the brain.

Conclusion: It seems the findings of this review can positively aid other researchers to develop medical guidelines to prevent or resolve the brain changes in central nervous structure and consequently cognitive impairments in children and adolescents.
\end{abstract}

\section{Background}

The relationship between diabetes and academic performance have been of great interest to researchers during the year to date. Many studies have been conducted to discover this relationship during three recent decades [1]. Also, other cognitive functions have been explored besides this relationship in the coming years including memory, attention and executive functions [2] which are some controversies in their relation with diabetes. However, very few studies have addresses the relationship between diabetes and cognitive impairment, academic performance, and its compliance with the structure of the nervous

\footnotetext{
* Correspondence: emrc@tums.ac.ir

${ }^{5}$ Endocrinology \& Metabolism Research Center, Endocrinology and Metabolism Clinical Sciences Institute, Tehran University of Medical Sciences, Shari'ati Hospital, North Kargar St., Tehran, Iran

Full list of author information is available at the end of the article
}

system [3]. In addition, the majority of these few studies are mostly conducted on older adults so that children and adolescents are thereby neglected.

However, with the development of functional imaging techniques in recent years, the changes in the performance of different areas of the central nervous system in children and adolescents with diabetes are evaluated with a greater precision [4]. But, evaluation of the structural changes of brain in the context of diabetes is of paramount importance. The detection of such relationship may offer an open window to identify the mechanism of cognitive changes in children and adolescents with diabetes and to design effective interventions to prevent these changes. The authors of the present paper are trying to prevent structural changes in children and adolescents living with diabetes using this pathway. 
Pediatric neuroimaging is faced by major challenges, among which one can name having no tendency of children for different imaging devices due to some fears [5]. On the other hand, despite various studies in this field, different ethical considerations come into play when implementing brain imaging in children and adolescents with diabetes as well as in healthy individuals [6].

Therefore, aiming at evaluating the relationship between diabetes and structural changes in the central nervous system in children and adolescents, the authors of this paper decided to conduct a systematic review to provide a response to the research question. By this systematic review and also considering executive and ethical issues, they hope there is no need to design and run further researches in order to evaluate the relation between diabetes and brain structural changes in children and adolescents.

For certain, finding the answers to these questions may be an effective step in alleviating the diabetes complications on the brain structure and subsequent cognitive functions in children and adolescents with diabetes.

\section{Methods}

This study is a systematic review conducted to investigate the structural changes in the central nervous system in children and adolescents living with diabetes.

\section{Search strategy}

In order to search the published research until the beginning of November 2016, the authors used all articles published in national and international journals indexed in the scientific databases of Pubmed and SCOPUS, as two large and prestigious sources of biomedical and life sciences journal literature. The articles were found using key words or their combinations in English including, neuroimaging, brain change, brain structural, brain map, and diabetes.

\section{Study selection}

The inclusion criteria used to select articles were publication in the last 10 years, publication in English, research on humans, sample aged between 0 and 18 years (in PubMed) and Category of children and adolescents (in SCOPUS).

A list was prepared by the researcher including the relevant titles and abstracts of all papers available in the above mentioned databases, and each were independently studied in order to select the relevant topics. Afterward, the repeated studies were eliminated followed by checking for titles, author(s) names, publication year, Number, and name of journal. Also, based on the titles of the articles, irrelevant unrelated articles to the subject of the present study were excluded from further assessment.

\section{Quality Assessment}

Followed by determining the relevant studies in terms of titles and abstracts, the researchers used STROBE checklist (i.e. strengthening the reporting of observational studies in epidemiology) which is a standard checklist to evaluate the selected papers. Articles given at least score 40 points according to the checklist questions were entered into the research, and their associated data were extracted for the systematic review.

\section{Data Extraction}

In order to extract data from the selected studies, the researchers evaluated them in terms of some details, including titles, first author's name, year of study, type of study, age group, sample size, type of imaging tool used, and the structural changes observed in the imaging

\section{Results}

\section{Search process}

Using relevant keywords and making a search with high sensitivity, 503 papers published until the beginning of November 2016 were identified. Of these articles, 58 articles were deleted due to duplication from the former evaluated banks. After the removal of titles unrelated to the subject of the study, 16 related articles remained. It should be noted that a large number of published articles on the subject of diabetes insipitus were excluded at this stage. For this reason, a considerable number of articles were excluded at this stage. Also, those articles examining the structural changes in the brain due to diabetic ketoacidosis, as one of the most important neurological complications in pediatric diabetes, were also excluded. Three other papers were eliminated due to lack of access to the full-text of article despite sending email to the correspondence author. Finally, 13 articles in the field of assessing structural changes in the central nervous system in children and adolescents with diabetes were entered into the evaluation process. The flowchart of search strategy and the evaluation of papers are presented in Fig. 1.

A summary of the key findings are as follows (Table 1):

\section{Evaluation of central nervous structures in patients with diabetes compared to healthy subjects}

In the majority of conducted studies so far, a group of children and adolescents with diabetes have been compared with a healthy peer where in the majority of them several differences in neural structures have been reported in patients with diabetes compared to the healthy individuals. In the study of Rofey et al. conducted on 15 male adolescents aged 12-21 years in three groups including, diagnosed with type 2 diabetes, obese nondiabetic, and normal- weight nondiabetic, it was found that the volume of caudate in the group with normal 


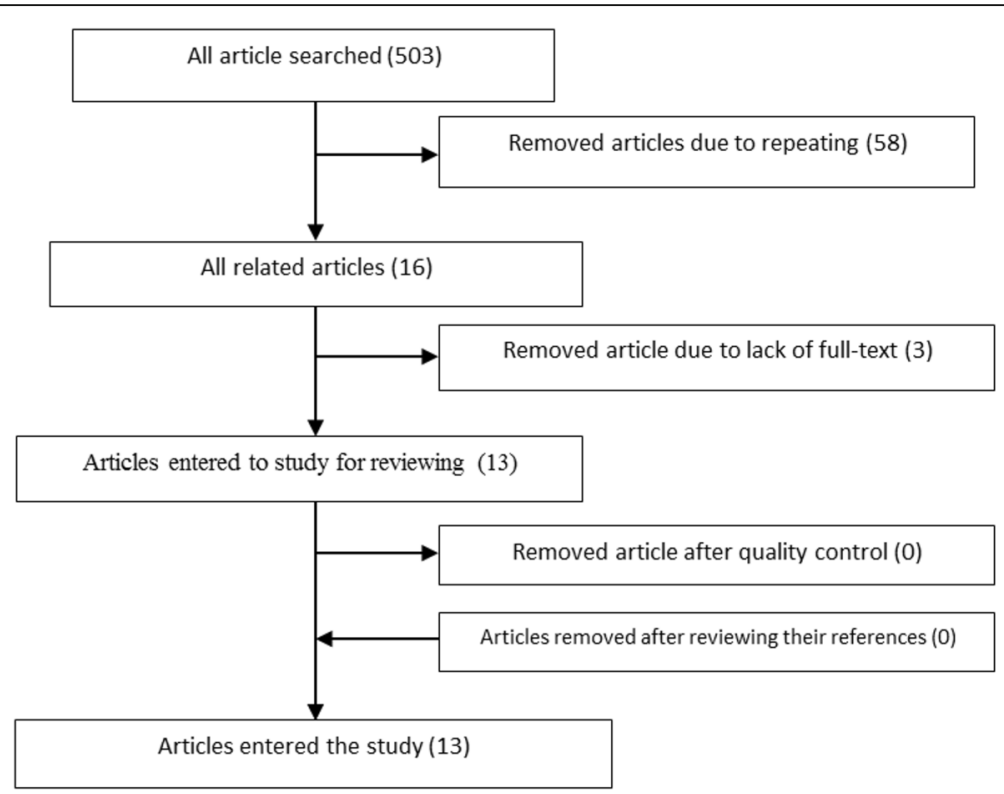

Fig. 1 Flowchart of search strategy and the evaluation of papers

weight was significantly higher compared to the obese and diabetic groups. Thalamic volumes in both normal and obese were higher in comparison with diabetics. Although not statistically significant, but such differences were found in some other regions, including the hippocampus, nucleus accumbens, amygdala, putamen, and pallidum. In evaluating white matter, diabetic group were reported to have the highest deficit in terms of functional anisotropy volume (FA). However, such differences were not significant [7].

In another study on 142 children with diabetes aged 4 to 10 years and 72 healthy subjects, Mauras et al. evaluated the structure of the central nervous system using MRI during two separate phases, at baseline and after 18 months. They drew the conclusion that children with diabetes showed a slower growth rate in parts of the gray and white matters volume compared to healthy subjects. Gray matter areas included left precuneus, right temporal, frontal and parietal lobes and right medialfrontal cortex, and white matter areas included splenium of corpus callosum, bilateral superior-pareital lobe, bilateral avterior forceps, and inferior-frontal fasciculus. After 18 months, diabetic children revealed significant differences compared to their healthy peers in the development of white and graymatters involved in the process of sensorimotor and cognition [8].

In another study on 142 children with diabetes and 68 healthy controls with an average age of 7 years (1.7), Marzelli et al. reported the reduced volume of gray matter in the bilateral occipital and cerebellar regions and increased volume of gray matter in left inferior prefrontal, insula, and temporal pole in children with diabetes compared to their health peers [9]. Also, in another study to assess the changes in white matter of brain in diabetic children, Barnea-Goraly et al. reported significantly reduced axial diffusivity (AD) in widespread brain regions [10].

In a similar study conducted by Antenor-Dorsey et al. to evaluate the microstructural changes of white matter in children with diabetes, lower fractional anisotropy in the superior parietal lobule and reduced mean diffusivity in the thalamus in the diabetic group were observed compared to healthy subjects [1]. Also, Aye et al. in their study on 22 diabetic children aged 3-10 years and 14 healthy subjects reported lower doses of axial diffusivity in temporal and parietal lobe regions: internal capsule, body of the corpus callosum, right singulategyrus and posterior parietal lobe in diabetic group than the controls. They also revealed significant differences in the fractional anisotropy and radial diffusivity in diabetic and healthy groups [11].

In another study, they also explored the structural differences in the brains of children with diabetes and evaluating 28 children with diabetes reported that after controlling for age and sex. The volume of white matter in diabetics didn't show the expected growth rate according to age compared with controls. The same trend was observed in hippocampal volume. But nothing was reported in the gray matter and the amygdala [12].

Developing a cohort study, Perantie et al. compared 75 diabetic children and adolescents with an average age of 12.5 years with 25 healthy subjects in terms of structural central nervous system at two time intervals in the framework of a two year study. Finally, they reported no 


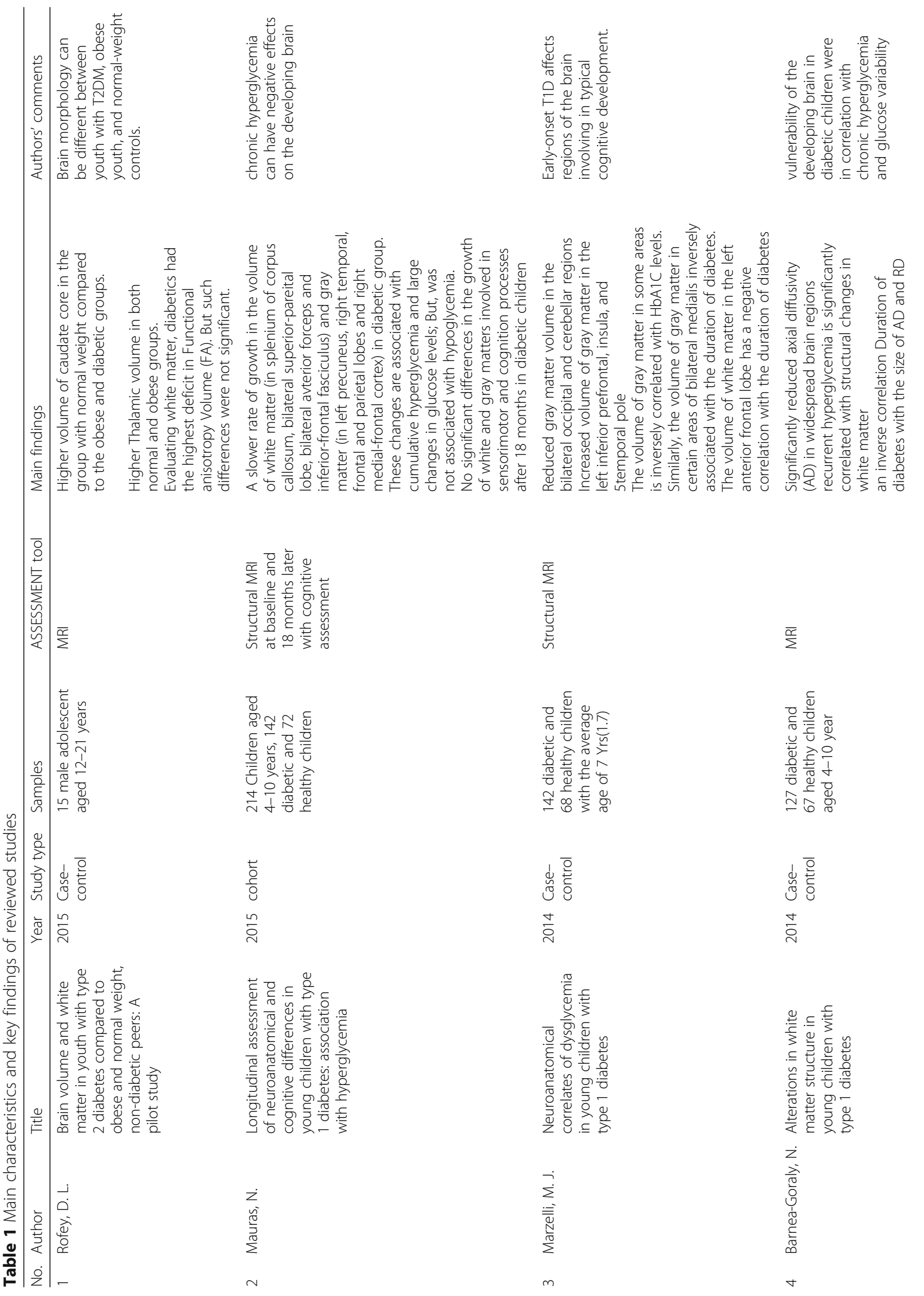



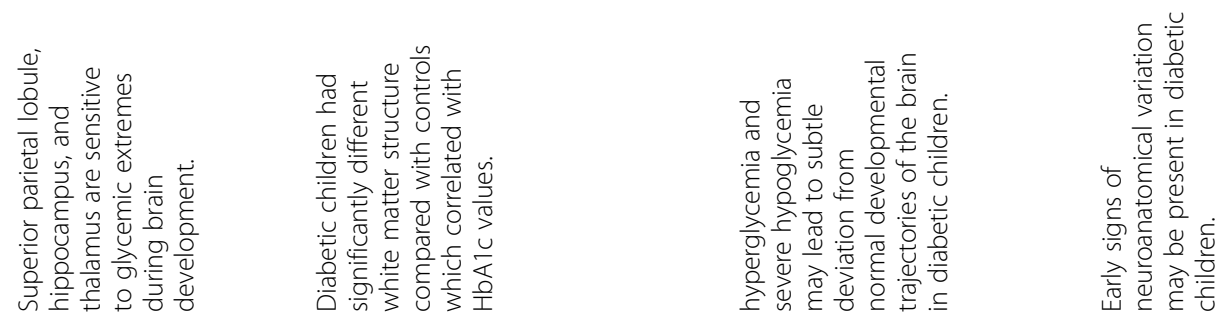

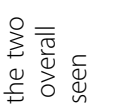
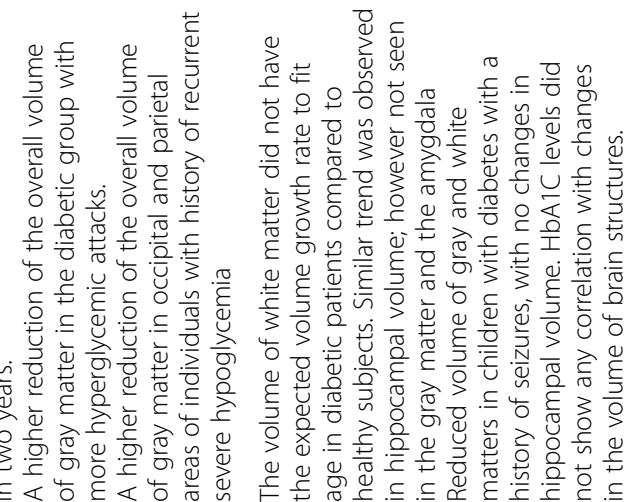

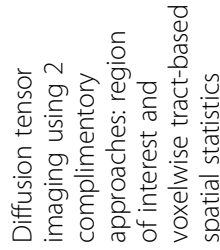

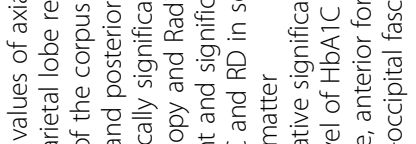

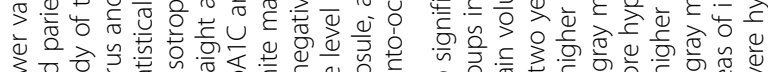

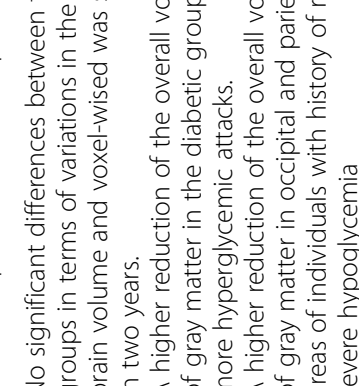

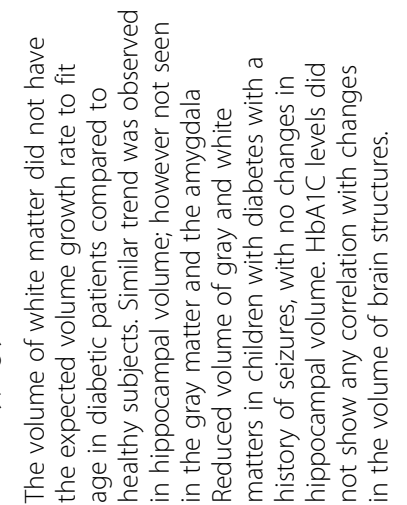

$\overline{\bar{\alpha}}$

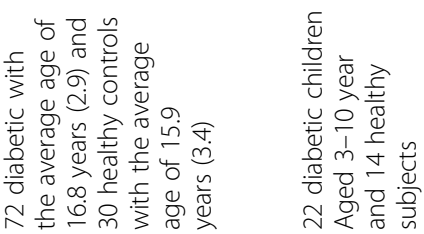

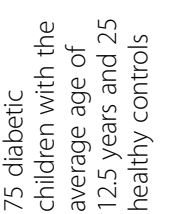

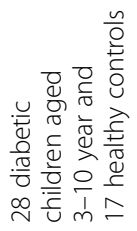

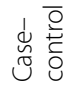

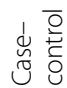

$\frac{5}{\frac{5}{0}}$

峁

i

ฉั

$\overline{\grave{~}}$

$\bar{\sigma}$

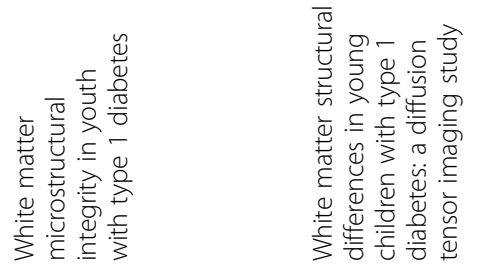

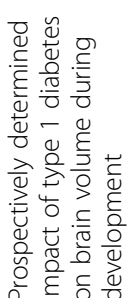

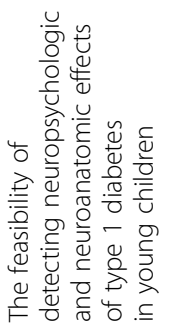

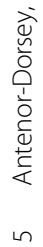

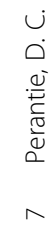

$\infty$ 


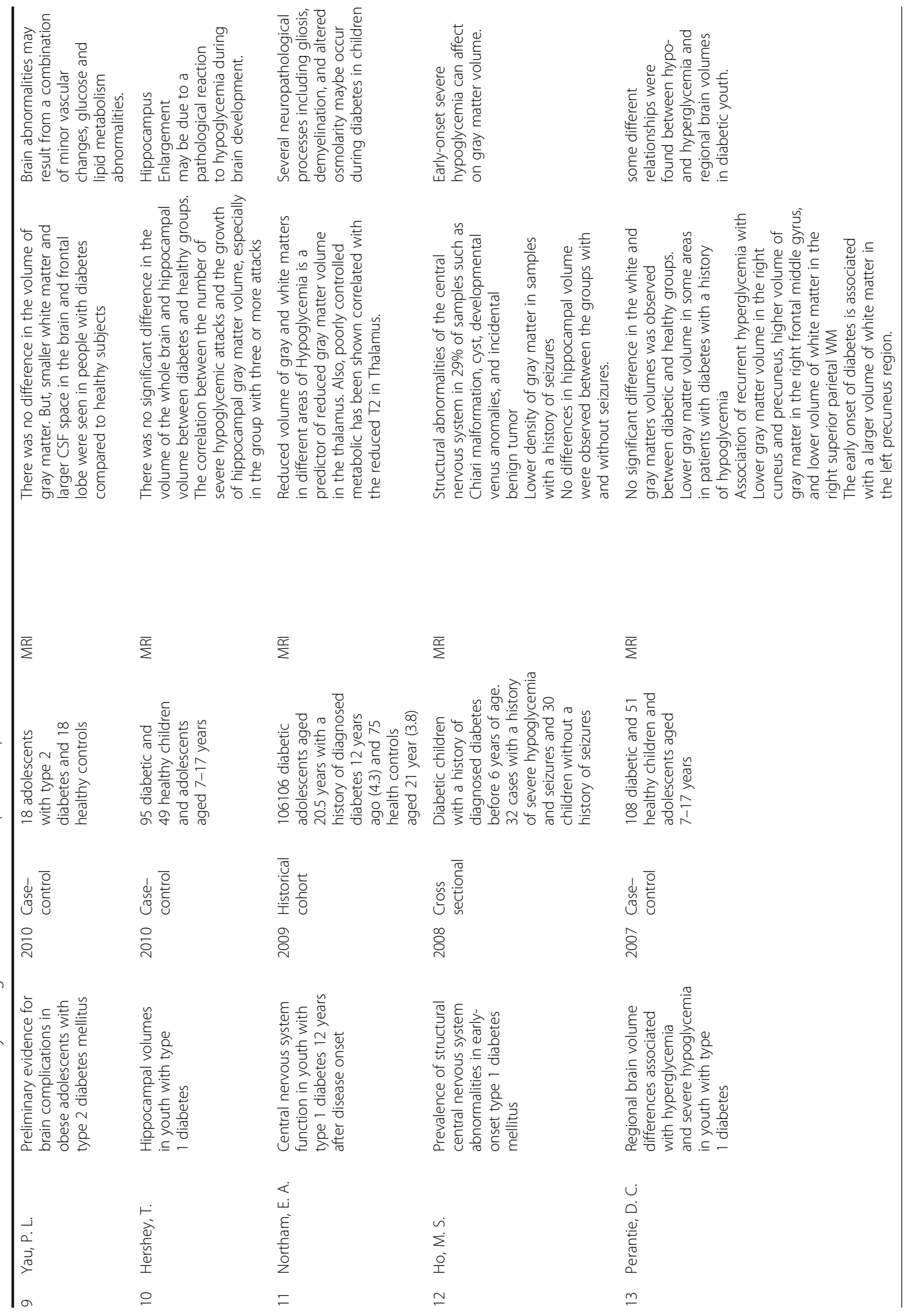


significant differences in the overall brain volume and voxel-wised changes between the groups in two years [13]. In another cross-sectional study on the diabetic children without evaluating changes over time, no significant difference between diabetic and healthy groups in the white and gray matters volume is reported [14].

In a different study, Yau et al. conducted a different study to analysis the brain structure of 18 obese adolescents with type 2 diabetes and 18 healthy obese adolescents in gray matter volume where no difference in the gray matter volume was observed. However, a smaller white matter, and larger CSF space was observed in the whole and in frontal lobe of people with diabetes compared to healthy subjects [15].

In addition, Hershey et al. in their study of diabetic children aged 7-17 years showed that after matching for the age and sex no significant difference in the whole brain and the hippocampus of diabetic and healthy children were observed [16]. The reduced volume of gray matter in bilateral thalami, right parahippocampal gyrus, right insular cortex and reduction of white matter volume in bilateral mesial temporal lobes (parahippocampal region), left temporal lobe, left middle frontal area in children and adolescents with diabetes compared to healthy subjects is demonstrated in the study of Northam et al.

This study was conducted on 106 diabetic patients aged 20.5 on the average diagnosed with diabetes since 12 years ago and 75 healthy subjects aged 21 (3.8) on the average. And in this respect, this research has important differences with other studies in which the longterm complications of diabetes on brain structures can be well demonstrated [17]. But, Ho et al. conducted a study on diabetic children diagnosed with diabetes before 6 years age aiming to assess the structural abnormalities in the central nervous system of children with diabetes. In this study, abnormalities in the central nervous system was observed in $29 \%$ of samples which included Chiari malformation, cyst, developmental venus anomaies, and incidental benign tumor. However, the prevalence of such abnormalities was not well compared with the health subjects group [18].

\section{Relationship between structural changes in the central nervous system and indicators of glycemic control}

In the majority of studies been made to evaluate the relationship between diabetes and the structural changes in the central nervous system its attempted to study the structural changes in the brain and the status of glycemic control in diabetic patient in addition to comparing diabetic children with healthy subjects. These studies, has put forward a clear illustration on the important facts regarding the role of glycemic control on the brain changes.
In their study, Mauras et al.along with assessing differences in brain structure among children with diabetes compared to healthy subjects, showed that these changes are related with cumulative hyperglycemia and notable changes in glucose levels; however, not correlated with hypoglycemia [8]. Marzelli et al. also showed that the volume of gray matter in areas such as bilateral lingual gyrus, fusiform gyrus, right parahippocampal gyrus, and cerebellum in are reversely associated with HbA1C levels.

Similarly, the volume of gray matter in areas of bilateral medial orbitofrontal, rectal gyri, and the anterior cingulate are inversely associated with the duration of diabetes. In addition, the volume of white matter in the left anterior frontal lobe has been shown to be negatively correlated with duration of disease [9]. Also, in the study of Barnea-Goraly et al. recurrent hyperglycemia in diabetic patients showed a significant correlation with structural changes in white matter. But, this correlation was not seen in cases of hypoglycemia.

Duration of diabetes revealed a significant inverse correlation with the size of axial diffusivity (AD) and radial diffusivity (RD) and a significant positive correlation with Fractional anisotropy (FA) values in widespread brain regions. No significant difference has been seen in the volume of white matter in children with diabetes who experience severe hypoglycemia and seizures and the rest of diabetics without such experiences. On the other hand, younger ages of developing diabetes showed a positive correlation with lower FA and higher RD [10]. In another study with a similar goal, a history of three or more severe attacks of hyperglycemia revealed a significant correlation with reduced anisotropy, increased diffusivity in the superior parietal lobule, and increased diffusivity in the hippocampus [19].

In the study of Aye et al., a direct and significant correlation between $\mathrm{HbA} 1 \mathrm{C}$ and $\mathrm{RD}$ was found which was observable in the inferior fronto -occipital fasciculi, uncinated fasciculi, subgenual WM, anterior forceps, right internal capsule, superior middle, inferior temporal gyri, splenium of corpus callusome, superior longitudinal fasciculi, and occipital WM. In this study, a negative correlation between the level of HbA1C and FA in right internal capsule, anterior forceps, inferior fronto-occipital fasciculi and splenium of CC have also been reported [11].

In another study, Aye et al. showed that diabetic children with a history of seizures developed a reduced volume of gray and white matters compared to those with diabetes who had no history of seizures. But, the history of seizures did not show any impact on the volume of the hippocampus. As well, HbA1C levels showed no correlation with changes in the volume of brain structures [12]. Also, in a two-year cohort study conducted on diabetic patients Perantie et al. concluded 
that patients with recurrent hyperglycemia episodes and a more intense reduced volume of gray matter. Moreover, patients who experience severe recurrent hypoglycemia were at higher risk of reducing the volume of white matter in areas of the occipital and parietal [13].

In another study on diabetic children, he also reported that patients with history of hypoglycemia had a lower volume of gray matter in the superior temporal, occipital cortex, and left inferior occipital cortex compared to the group without a history of hypoglycemia.

In diabetes, recurrent hyperglycemia was found to be associated with less gray matter volume in the right cuneus and precuneus. As well, it was associated with higher volume of gray matter in the right frontal middle gyrus and lower volume of white matter in the right superior parietal (WM). Also, its indicated that the early onset of the disease was related with greater white matter volume in the left precuneus region [14]. In the study of Hershey et al., the number of severe hypoglycemic attacks was significantly correlated with the enlargement of hippocampal gray matter volume. This correlation was more highlighted in the subgroup with 3 or more severe attacks. This study didn't find any pattern indicating the changes in the hippocampal gray matter volume due to chronic hyperglycemia [16].

Northamet al. also claimed in their research that hypoglycemia is a predictor of gray matter loss in the thalamus. This survey also revealed an association between poorly controlled metabolic and $\mathrm{T} 2$ reduction in the thalamus [17].

\section{Discussion and conclusion}

Several evidence is available indicating the structural changes in the central nervous system as a complication of diabetes in adults [20]. Also, in some studies, vascular complications in the context of diabetes is considered as one of the factors contributing to brain changes in patients with diabetes which can increase the risk of some disorders such as stroke [21]. Incidentally, disorders such as dementia, as one of the neurological complications of diabetes, are linked to this problem [22]. But, the analysis of structural changes in the brains of diabetic children is a subject that has been discussed in recent years and various studies have been conducted in this regard. However, as may be expected fewer literature is available on the brain structure complications in pediatric diabetes compared to in adults.

The authors of the present study have attempted to make a comprehensive review of the efforts of other researchers to explore the association between diabetes and brain structural changes. Of course, neurological changes in the context of some severe complications of diabetes, such as ketoacidosis have been the subject of several studies which were not included in this study. This is mainly because the subjects in these studies were merely children with diabetes who had these complications and may not be a perfect example of the diabetic children population.

As can be seen in these studies, a huge proportion of structures of the central nervous system have been affected by diabetes that include different areas of gray and white matters. Of these studies, only two longitudinal studies have evaluated the developmental changes in the central nervous system in diabetic patients over times, which have not reported consistent results with together. Other studies reviewed in this paper are cross-sectional and case-control studies which the majority have reported various brain structural differences in children and adolescents with diabetes compare to healthy subjects.

This could explain the cognitive changes in diabetic children who have been investigated in several studies. Also, newer researches on children and adolescents with diabetes conducted by the application of functional brain imaging techniques have presented new evidence regarding this association.

Of other important aspects that should be taken into account in these studies is the association of glycemic control and the structural changes in the central nervous system of children and adolescents with diabetes. In the majority of these studies, it has become clear that high glycemic changes, especially recurrent hyperglycemic attacks are very seriously associated with structural changes in the brain.

Now, if one takes into consideration the fact that a significant proportion of children and adolescents with diabetes lack good glycemic control parameters [23], it must be accepted that many children and adolescents with diabetes should suffer from structural changes in the brain and, consequently, serious cognitive changes during the course of their disease.

This issue must be well taken into account and the families of children with diabetes must be well understood by the health care providers.

One of the considerations that must be made when reviewing these studies is that apart from two studies on children and adolescents with type 2 diabetes, other studies had been conducted on patients with type 1 diabetes. Moreover, none of the studies have compared the changes in brain structure in children and adolescents with type 1 diabetes and those with type 2 , and in this respect, the difference between these two types of diseases affecting the central nervous system is not well illustrated. On the other hand, given the growing trend of developing type 2 diabetes among adolescents, further evidence indicating the association between type 2 diabetes and the structural changes of brain is needed [24].

Of the limitations observable in related studies are the lack of prospective studies exploring this association. Surely, design and implementation of prospective studies can provide answers to serious questions in this area 
including whether adequate glycemic control after the structural changes of the nervous system can resolve the changes or not, and or the brain structural changes seen in children and adolescents with diabetes compared to healthy peers is merely due to diabetes or other effective factors have created such differences. The answers to these questions can positively aid therapists to develop medical guidelines to prevent or resolve the changes in nervous structure.

\section{Abbreviations}

AD: axial diffusivity; CC: corpus callosum; CSF: Cerebro Spinal Fluid; FA: Functional anisotropy; MRI: Magnetic Resonance Imaging; RD: Radial diffusivity; WM: White Matter

\section{Acknowledgment}

We would like to thank Jalal Farzami for his help in preparing this article.

\section{Funding}

Not applicable.

\section{Availability of data and materials}

The data of publications gathered during the current study are available from the corresponding author on reasonable request.

\section{Author contributions}

AP.: conception and study design, gathering data, drafting the manuscript MT-D.: conception and study design, revising draft critically for important intellectual content. SEQ:: conception and study design, revising draft critically for important intellectual content. MA.: revising draft critically for important intellectual content. BL: conception and study design, accountable for all aspects of the work in ensuring that questions related to the accuracy or integrity of any part of the work are appropriately investigated and resolved.

\section{Competing interests}

The authors declare that they have no competing interests.

\section{Consent for publication}

Not applicable.

\section{Ethics approval and consent to participate}

This review article is one of the outputs of the project entitled "Evaluation of the Correlation Between Type 1 Diabetes and Cognitive Function in Children and Adolescents, and the Synchronization of This Correlation with Structural Changes in the Central Nervous System" which has been approved by ethics committee of EMRC of Tehran University of Medical sciences (TUMS) with reference number 00300

\section{Author details}

'Diabetes Research Center, Endocrinology and Metabolism Clinical Sciences Institute, Tehran University of Medical Sciences, Tehran, Iran. ${ }^{2}$ Department of Psychiatry, Rouzbeh Hospital, Tehran University of Medical Sciences, Tehran, Iran. ${ }^{3}$ Faculty of psychology and education, Allameh Tabataba'ee university, Tehran, Iran. ${ }^{4}$ Elderly Health Research Center, Endocrinology and Metabolism Population Sciences Institute, Tehran University of Medical Sciences, Tehran, Iran. ${ }^{5}$ Endocrinology \& Metabolism Research Center, Endocrinology and Metabolism Clinical Sciences Institute, Tehran University of Medical Sciences, Shari'ati Hospital, North Kargar St., Tehran, Iran.

Received: 8 January 2017 Accepted: 10 February 2017

Published online: 03 March 2017

\section{References}

1. Galatzer A, Amir S, Gil R, Karp M, Laron Z. Crisis intervention program in newly diagnosed diabetic children. Diabetes Care. 1982:5(4):414-9.

2. Kirchhoff BA, Jundt DK, Doty T, Hershey T. A longitudinal investigation of cognitive function in children and adolescents with type 1 diabetes mellitus. Pediatr Diabetes. 2016. doi:10.1111/pedi.12414
3. Pourabbasi A, Tehrani-Doost M, Ebrahimi Qavam S, Larijani B. Evaluation of the correlation between type 1 diabetes and cognitive function in children and adolescents, and comparisonof this correlation with structural changes in the central nervous system: a study protocol. BMJ Open. 2016;6(4):e007917.

4. Cameron FJ. The Impact of Diabetes on Brain Function in Childhood and Adolescence. Pediatr Clin N Am. 2015;62(4):911-27.

5. Staphorst MS, Hunfeld JA, van de Vathorst S, Passchier J, van Goudoever JB. Children's self reported discomforts as participants in clinical research. Soc Sci Med. 2015:142:154-62.

6. Hinton VJ. Ethics of neuroimaging in pediatric development. Brain Cogn. 2002:50(3):455-68.

7. Rofey DL, Arslanian SA, El Nokali NE, Verstynen T, Watt JC, Black JJ, et al. Brain volume and white matter in youth with type 2 diabetes compared to obese and normal weight, non-diabetic peers: A pilot study. Int J Dev . 2015:46:88-91.

8. Mauras N, Mazaika P, Buckingham B, Weinzimer S, White NH, Tsalikian E, et al. Longitudinal assessment of neuroanatomical and cognitive differences in young children with type 1 diabetes: association with hyperglycemia. Diabetes. 2015;64(5):1770-9.

9. Marzelli MJ, Mazaika PK, Barnea-Goraly N, Hershey T, Tsalikian E, Tamborlane W, et al. Neuroanatomical correlates of dysglycemia in young children with type 1 diabetes. Diabetes. 2014;63(1):343-53.

10. Barnea-Goraly N, Raman M, Mazaika P, Marzelli M, Hershey T, Weinzimer SA, et al. Alterations in white matter structure in young children with type 1 diabetes. Diabetes Care. 2014;37(2):332-40.

11. Aye T, Barnea-Goraly N, Ambler C, Hoang S, Schleifer $K$, Park Y, et al. White matter structural differences in young children with type 1 diabetes: a diffusion tensor imaging study. Diabetes Care. 2012;35(11):2167-73.

12. Aye $T$, Reiss AL, Kesler S, Hoang S, Drobny J, Park Y, et al. The feasibility of detecting neuropsychologic and neuroanatomic effects of type 1 diabetes in young children. Diabetes Care. 2011;34(7):1458-62.

13. Perantie DC, Koller JM, Weaver PM, Lugar HM, Black KJ, White NH, et al. Prospectively determined impact of type 1 diabetes on brain volume during development. Diabetes. 2011;60(11):3006-14

14. Perantie DC, Wu J, Koller JM, Lim A, Warren SL, Black KJ, et al. Regional brain volume differences associated with hyperglycemia and severe hypoglycemia inyouth with type 1 diabetes. Diabetes Care. 2007:30(9):2331-7.

15. Yau PL, Javier DC, Ryan CM, Tsui WH, Ardekani BA, Ten S, et al. Preliminary evidence for brain complications in obese adolescents with type 2 diabetes mellitus. Diabetologia. 2010;53(11):2298-306.

16. Hershey T, Perantie DC, Wu J, Weaver PM, Black KJ, White NH. Hippocampal volumes in youth with type 1 diabetes. Diabetes. 2010;59(1):236-41.

17. Northam EA, Rankins D, Lin A, Wellard RM, Pell GS, Finch SJ, et al. Central nervous system function in youth with type 1 diabetes 12 years after disease onset. Diabetes Care. 2009;32(3):445-50.

18. Ho MS, Weller NJ, Ives FJ, Carne CL, Murray K, Vanden Driesen Rl, et al. Prevalence of structural central nervous system abnormalities in early-onset type 1 diabetes mellitus. J Pediatr. 2008;153(3):385-90.

19. Antenor-Dorsey JA, Meyer E, Rutlin J, Perantie DC, White NH, Arbelaez AM, et al. White matter microstructural integrity in youth with type 1 diabetes. Diabetes. 2013;62(2):581-9.

20. Seaquist ER. The Impact of Diabetes on Cerebral Structure and Function. Psychosom Med. 2015;77(6):616-21.

21. Kozakova M, Palombo C. Diabetes Mellitus, Arterial Wall, and Cardiovascular Risk Assessment. Int J Environ Res Public Health. 2016;13(2):201.

22. Sherzai D, Sherzai A, Lui K, Pan D, Chiou D, Bazargan M, et al. The Association Between Diabetes and Dementia Among Elderly Individuals: A Nationwide Inpatient Sample Analysis. J Geriatr Psychiatry Neurol. 2016; 29(3):120-5

23. Hood KK, Beavers DP, Yi-Frazier J, Bell R, Dabelea D, McKeown RE, et al. Psychosocial burden and glycemic control during the first 6 years of diabetes: results from the SEARCH for Diabetes in Youth study. J Adolesc. 2014;55(4):498-504.

24. Lee JH, Kim YM, Kwak MJ, Kim SY, Kim HJ, Cheon CK, et al. Incidence trends and associated factors of diabetes mellitus in Korean children and adolescents: a retrospective cohort study in Busan and Gyeongnam. Annals of pediatric endocrinology \& metabolism. 2015;20(4):206-12. 